\title{
Clot-bound Thrombin Is Protected from Inhibition by Heparin-Antithrombin III but Is Susceptible to Inactivation by Antithrombin III-independent Inhibitors
}

\author{
Jeffrey I. Weitz, Monika Hudoba, David Massel, John Maraganore, and Jack Hirsh \\ Department of Medicine, McMaster University and the Hamilton Civic Hospitals Research Center, \\ Hamilton, Ontario, L8V 1C3, Canada; and Biogen Inc., Cambridge, Massachusetts 02142
}

\section{Abstract}

Propagation of venous thrombi or rethrombosis after coronary thrombolytic therapy can occur despite heparin administration. To explore potential mechanisms, we set out to determine whether clot-bound thrombin is relatively protected from inhibition by heparin-antithrombin III but susceptible to inactivation by antithrombin III-independent inhibitors. Using plasma fibrinopeptide A (FPA) levels as an index of thrombin activity, we compared the ability of thrombin inhibitors to block FPA release mediated by fluid-phase thrombin with their activity against the clot-bound enzyme. Incubation of thrombin with citrated plasma results in concentration-dependent FPA generation, which reaches a plateau within minutes. In contrast, there is progressive FPA generation when fibrin clots are incubated with citrated plasma. Heparin, hirudin, hirudin dodecapeptide (hirugen), and D-phenylalanyl-L-prolyl-L-arginyl chloromethyl ketone (PPACK) produce concentration-dependent inhibition of FPA release mediated by fluid-phase thrombin. However, heparin is much less effective at inhibiting thrombin bound to fibrin because a 20 -fold higher concentration is necessary to block $70 \%$ of the activity of the clot-bound enzyme than is required for equivalent inhibition of fluid-phase thrombin ( 2.0 and $0.1 \mathrm{U} / \mathrm{ml}$, respectively). In contrast, hirugen and PPACK are equally effective inhibitors of fluid- and solidphase thrombin, while hirudin is only $50 \%$ as effective against the clot-bound enzyme. None of the inhibitors displace bound ${ }^{125}$ I-labeled thrombin from the clot. These studies indicate that (a) clot-bound thrombin is relatively protected from inhibition by heparin, possibly because the heparin binding site on thrombin is inaccessible when the enzyme is bound to fibrin, and $(b)$ clot-bound thrombin is susceptible to inactivation by antithrombin III-independent inhibitors because the sites of their interaction are not masked by thrombin binding to fibrin. For these reasons, antithrombin III-independent inhibitors may be more effective than heparin in certain clinical settings. (J. Clin. Invest. 1990. 86:385-391.) Key words: thrombin • fibrin • heparin • antithrombin III

This work was presented in part at the 62nd Scientific Sessions of the American Heart Association (1989. Circulation. 80:420a. [Abstr.]) and the 31st Annual Meeting of the American Society of Hematology (1989. Blood. 74:136a. [Abstr.]).

Address correspondence to Dr. Jeffrey Weitz, Henderson General Hospital, 711 Concession Street, Hamilton, Ontario, L8V 1C3, Canada.

Received for publication 27 September 1989 and in revised form 21 February 1990.

J. Clin. Invest.

(c) The American Society for Clinical Investigation, Inc.

0021-9738/90/08/0385/07 \$2.00

Volume 86, August 1990, 385-391

\section{Introduction}

Thrombin converts fibrinogen into insoluble fibrin by cleaving fibrinopeptides A and B (FPA and FPB, respectively) ${ }^{1}$ from the amino-terminal regions of the $\mathrm{A} \alpha$ and $\mathrm{B} \beta$ chains of fibrinogen, respectively (1). During this reaction thrombin is adsorbed to fibrin through a site distinct from its catalytic serine center (2-7). Since thrombin bound to fibrin equilibrates slowly with the external solution (3), its reactivity with inhibitors outside the clot may be limited.

Heparin is an effective antithrombotic agent that acts primarily by catalyzing the inactivation of thrombin and activated Factor $X$ by antithrombin III (8-11). Recent studies done in buffer systems have demonstrated that thrombin bound to fibrin monomer is resistant to inactivation by heparin-antithrombin III (12). This phenomenon may explain why heparin is limited in its ability to inhibit the propagation of venous thrombi, and to prevent rethrombosis after successful coronary thrombolysis. In experimental animals, fibrin accretion onto jugular vein thrombi is only $50 \%$ inhibited by high concentrations of heparin (13). Similarly, propagation of thrombi in the deep veins of the lower extremities occurs in $\sim 10 \%$ of patients during heparin therapy (14-16). Further, unless maintenance anticoagulant therapy is given to patients with deep vein thrombosis, clot extension occurs in $29-47 \%$ of patients treated with a 5-14-d course of heparin $(17,18)$. Finally, heparin does not prevent rethrombosis after successful tissue plasminogen activator-induced coronary thrombolysis in experimental animals (19) or in man (20).

The limitations of heparin therapy are consistent with the hypothesis that in a plasma system, thrombin bound to fibrin is protected from inactivation by heparin-antithrombin III. Accordingly, we have performed studies in plasma to determine whether thrombin bound to fibrin is $(a)$ enzymatically active, $(b)$ relatively resistant to inhibition by heparin-antithrombin III, and $(c)$ more susceptible to inactivation by antithrombin III-independent inhibitors.

\section{Methods}

Reagents. Human $\alpha$-thrombin, sp act $2,850 \mathrm{U} / \mathrm{mg}$, was generously provided by Dr. J. Fenton II, New York State Department of Health, Albany, NY. Standard heparin from porcine intestinal mucosa (grade II) was purchased from Sigma Chemical Co., St. Louis, MO, while D-phenylalanyl-L-prolyl-L-arginyl chloromethyl ketone (PPACK) was from Calbiochem Corp., San Diego, CA. Recombinant hirudin with a Val-Val amino-terminal sequence was obtained from American Diagnostica, New York, NY. The synthetic, tyrosine-sulfated dodecapep-

1. Abbreviations used in this paper: FPA, fibrinopeptide A; PPACK, D-phenylalanyl-L-prolyl-L-arginyl chloromethyl ketone; TBS, $0.1 \mathrm{M}$ $\mathrm{NaCl}$ buffered with $0.05 \mathrm{M}$ Tris $\mathrm{HCl}, \mathrm{pH}$ 7.4. 
tide hirugen (Biogen Inc., Cambridge, MA), comprising residues 53-64 of hirudin (H-peptide, BG 8865) was prepared by solid-phase peptide synthesis, chemical sulfation of Tyr-11, and final purification using reverse-phase HPLC (21). By forming a 1:1 complex with thrombin, hirugen inhibits the fibrinogenolytic activity of the enzyme but does not impair its amidolytic activity. Based on these findings, it is likely that hirugen interacts with a noncatalytic site on thrombin (21).

Radioimmunoassay of FPA. FPA was assayed as previously described (22) using antiserum R2. This antibody is specific for FPA (23, 24) and does not crossreact with fibrinogen or FPA-containing fragments derived from the amino-terminal region of the $A \alpha$ chain of fibrinogen (24-26).

Thrombin-mediated FPA release from fibrinogen in plasma. Using a 21-gauge butterfly needle, blood was collected from the antecubital veins of healthy volunteers into plastic syringes prefilled with 1:10 vol of $3.8 \%$ trisodium citrate. After thorough mixing with the anticoagulant, the red cells were sedimented by centrifugation at $1,700 \mathrm{~g}$ for 15 $\min$ at $4^{\circ} \mathrm{C}$, and the platelet-poor plasma was harvested.

Human $\alpha$-thrombin, in concentrations ranging from 0.02 to 0.4 $\mathrm{nM}$, was incubated with $500-\mu \mathrm{l}$ aliquots of citrated plasma for $60 \mathrm{~min}$ at $37^{\circ} \mathrm{C}$ in the presence or absence of varying concentrations of the following thrombin inhibitors: heparin, hirudin, hirugen, and PPACK. At intervals, $100-\mu 1$ aliquots were removed and the reaction was terminated and fibrinogen was precipitated by the addition of $300 \mu \mathrm{l}$ of chilled ethanol followed by centrifugation at $15,000 \mathrm{~g}$ for $5 \mathrm{~min}$. The ethanol supernatants were then evaporated to dryness in a Speed-Vac concentrator (Savant Instruments, Inc., Hicksville, NY), reconstituted to original volume with distilled water, and assayed for FPA.

Preparation of ${ }^{125}$ I-labeled fibrinogen. Fibrinogen was precipitated from barium sulfate adsorbed plasma with $2 \mathrm{M} \beta$-alanine as described elsewhere (27). The isolated fibrinogen was then trace-labeled with ${ }^{125} I$ by the method of McFarlane (28) to a sp act of $100 \pm 5 \mu \mathrm{Ci} / \mathrm{mg}$.

Formation of ${ }^{125}$ I-labeled fibrin clots. Fresh citrated plasma was supplemented with ${ }^{125} \mathrm{I}$-labeled fibrinogen $(\sim 120,000 \mathrm{cpm} / \mathrm{ml})$ and 500- $\mu$ l aliquots were then transferred to polypropylene Eppendorf tubes. ${ }^{125} \mathrm{I}$-Labeled fibrin clots were formed around wire hooks by the addition of $\mathrm{CaCl}_{2}$ (final concentration, $25 \mathrm{mM}$ ). For some experiments, ${ }^{125}$ I-labeled clots of varying sizes were prepared by the recalcifcation of plasma in volumes ranging from $250 \mu \mathrm{l}$ to $1 \mathrm{ml}$. In all cases, however, the clots were aged for $60 \mathrm{~min}$ at $37^{\circ} \mathrm{C}$ with constant agitation, and then washed 10 times with $1-\mathrm{ml}$ aliquots of $0.1 \mathrm{M} \mathrm{NaCl}$ buffered with $0.05 \mathrm{M}$ Tris-HCl, pH 7.4 (TBS), over the course of $18 \mathrm{~h}$ to eliminate FPA trapped within the clots. To determine the efficiency of washing, aliquots taken from each of the washes were assayed for FPA. The washed clots were then counted for radioactivity for $1 \mathrm{~min}$ using a gamma counter (LKB Instruments, Inc., Gaithersburg, MD).

FPA generation by thrombin bound to fibrin. Washed ${ }^{125}$ I-labeled fibrin clots were incubated in 1-ml aliquots of fresh citrated plasma for $60 \mathrm{~min}$ at $37^{\circ} \mathrm{C}$ in the presence or absence of the various thrombin inhibitors. In control experiments, clots were incubated in TBS in place of plasma. At intervals, $100-\mu \mathrm{l}$ aliquots were removed and the unreacted fibrinogen was precipitated by the addition of $300 \mu \mathrm{l}$ chilled ethanol followed by centrifugation at $15,000 \mathrm{~g}$ for $5 \mathrm{~min}$. The ethanol supernatants were then evaporated to dryness in a Speed-Vac concentrator, reconstituted to original volume with distilled water, and assayed for FPA.

Preparation of ${ }^{125}$ I-labeled thrombin. Human $\alpha$-thrombin was iodinated by the solid-phase lactoperoxidase-glucose oxidase procedure (Enzymobead reagent; Bio-Rad Laboratories, Mississauga, Ontario, Canada) as described by Martin et al. (29). The ${ }^{125}$ I-labeled thrombin had a sp act of $\sim 5.4 \times 10^{4} \mu \mathrm{Ci} / \mathrm{mg}$, and was indistinguishable from native thrombin by SDS-PAGE and by its ability to clot fibrinogen.

Binding of ${ }^{125}$ I-labeled thrombin to fibrin. ${ }^{125} \mathrm{I}$-Labeled thrombin was added to citrated plasma $(\sim 100,000 \mathrm{cpm} / \mathrm{ml})$, and $500-\mu \mathrm{l}$ aliquots of plasma were then transferred to polypropylene Eppendorf tubes. Clots were formed around wire hooks as described above. After incubation for $60 \mathrm{~min}$ at $37^{\circ} \mathrm{C}$ the clots were removed and washed three times with $500-\mu 1$ aliquots of TBS, and the incorporation of
${ }^{125}$ I-labeled thrombin was determined by counting the clots for radioactivity for $1 \mathrm{~min}$. The clots were then washed extensively for $18 \mathrm{~h}$ as described above, and counted again for radioactivity to quantify the residual ${ }^{125} \mathrm{I}$-labeled thrombin bound to fibrin.

Effects of thrombin inhibitors on ${ }^{125}$ I-labeled thrombin bound to fibrin. Clots were incubated at $37^{\circ} \mathrm{C}$ in $500-\mu 1$ aliquots of plasma or buffer in the presence or absence of the various thrombin inhibitors. At 30- and 60-min incubation, 20- $\mu$ l aliquots were collected and counted for radioactivity to monitor release of ${ }^{125}$ I-labeled thrombin. At the same times, 100- $\mu$ l aliquots also were removed and the unreacted fibrinogen was precipitated with $300 \mu$ l of chilled ethanol followed by centrifugation at $15,000 \mathrm{~g}$ for $5 \mathrm{~min}$. The ethanol supernatants were then evaporated to dryness, reconstituted to original volume with distilled water, and assayed for FPA. At the end of the 60-min incubation period the clots were removed, washed three times with $500-\mu 1$ aliquots of TBS, and then counted to quantify the residual ${ }^{125}$ I-labeled thrombin bound to fibrin.

\section{Results}

Thrombin-mediated FPA release from fibrinogen in plasma. The incubation of thrombin with citrated plasma results in concentration-dependent generation of FPA which rapidly reaches a plateau as the enzyme is neutralized by antithrombins (Fig. 1). By plotting the amount of FPA released at 60min incubation as a function of the thrombin concentration, a dose-response curve can be constructed (Fig. 2).

Effect of thrombin inhibitors on thrombin-mediated FPA release. Using the plasma system, we compared the ability of each of the thrombin inhibitors to block FPA release mediated by fluid-phase thrombin at concentrations ranging from 0.02 to $0.4 \mathrm{nM}$. As illustrated in Fig. 2, each agent produces concentration-dependent inhibition of thrombin-induced FPA release.

Formation of ${ }^{125}$ I-labeled fibrin clots. The recalcification of $500-\mu 1$ aliquots of plasma containing ${ }^{125} \mathrm{I}$-labeled fibrinogen results in the formation of fibrin clots of standard size as determined by their radioactivity. Over $95 \%$ of the radiolabeled fibrinogen is incorporated into the clot so that the radioactivity is $57,342 \pm 1,280 \mathrm{cpm}$ (mean $\pm \mathrm{SD})$.

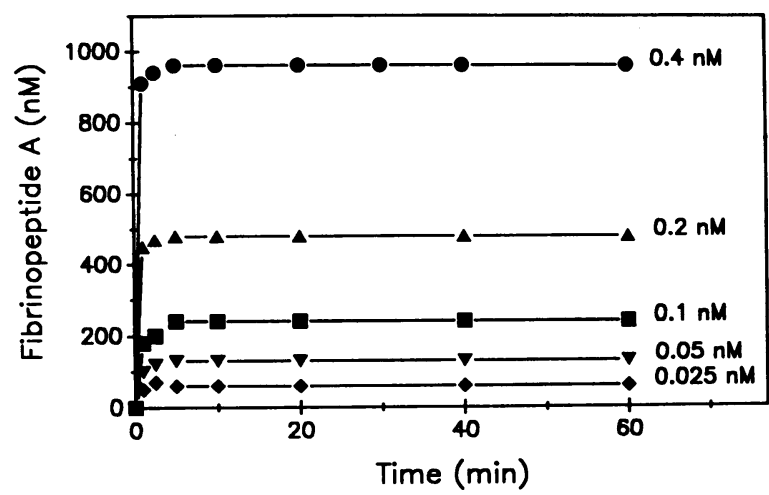

Figure 1. Time course of thrombin-mediated FPA generation in plasma. Human $\alpha$-thrombin, in the concentrations shown, was incubated within citrated plasma for $60 \mathrm{~min}$ at $37^{\circ} \mathrm{C}$. At the times indicated, aliquots were removed, unreacted fibrinogen was precipitated with ethanol, and the ethanol supernatants were assayed for FPA. Each point represents the mean of three separate experiments, each done in duplicate. 


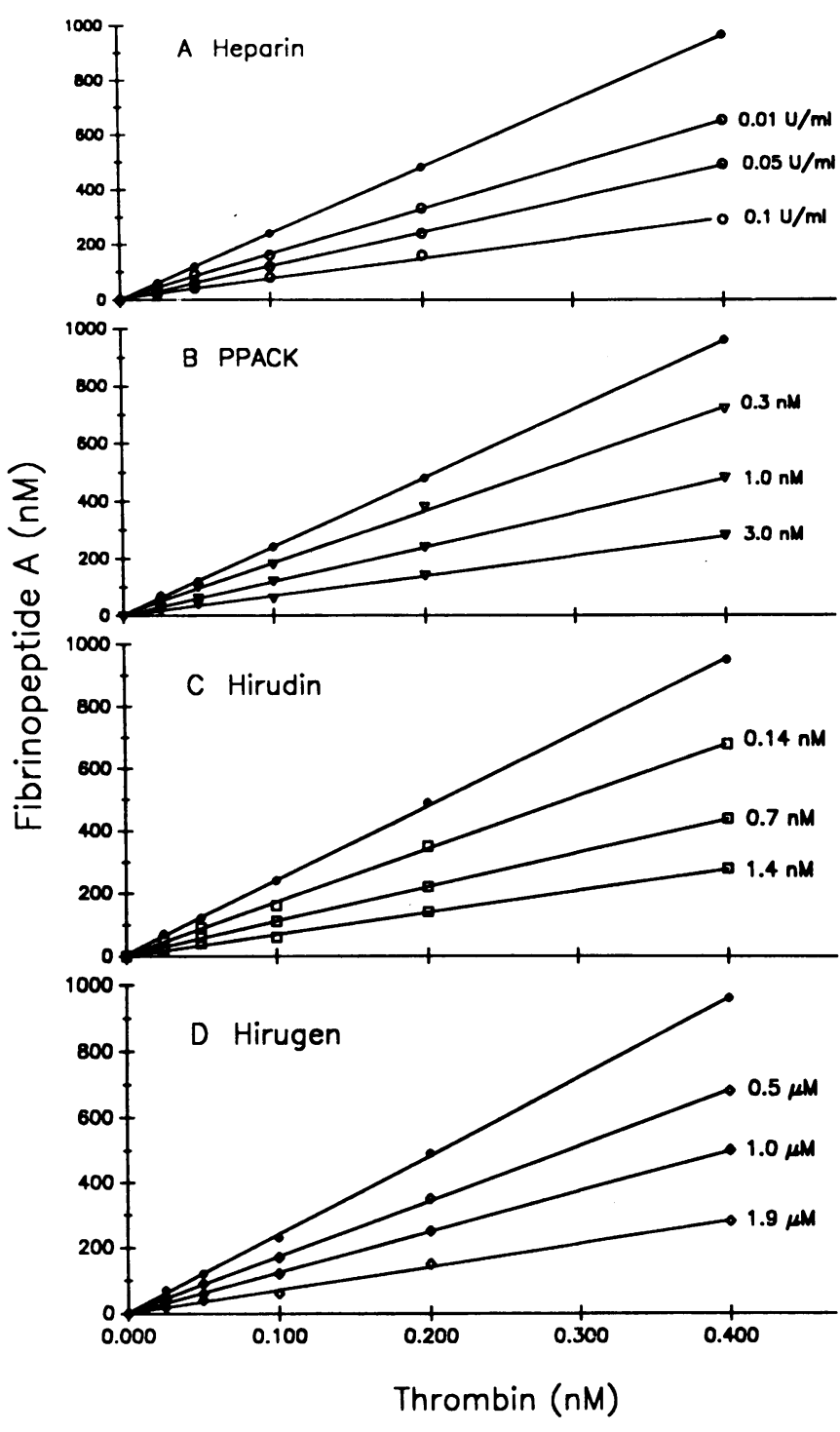

Figure 2. Effect of thrombin inhibitors on thrombin-mediated FPA release. Human $\alpha$-thrombin, in the concentrations shown, was incubated with citrated plasma for $60 \mathrm{~min}$ at $37^{\circ} \mathrm{C}$ in the absence (•) or presence of varying concentrations of the following thrombin inhibitors: $A$, heparin (o); $B$, PPACK ( $\nabla) ; C$, hirudin ( $\square)$; and $D$, hirugen $(\diamond)$. At the end of the incubation period, unreacted fibrinogen was precipitated with ethanol and the ethanol supernatants were assayed for FPA. Each point represents the mean of three separate experiments.

Extensive washing of the clots is essential to remove trapped FPA. Assay of FPA in successive washes demonstrates that most of the FPA is removed in the first $4 \mathrm{~h}$. To ensure complete removal of trapped FPA, however, the clots were washed for $18 \mathrm{~h}$. At this time $<5 \mathrm{nM}$ FPA was recovered in the wash buffer.

FPA generation by clot-bound thrombin. When washed ${ }^{125}$ I-labeled fibrin clots are incubated in citrated plasma, there is time-dependent generation of FPA (Fig. 3). In contrast, no increase in the plasma FPA level occurs when plasma is incubated in the absence of a clot (Fig. 3). To confirm that clotbound thrombin is responsible for the increase in the FPA levels, several control experiments were performed. First, this

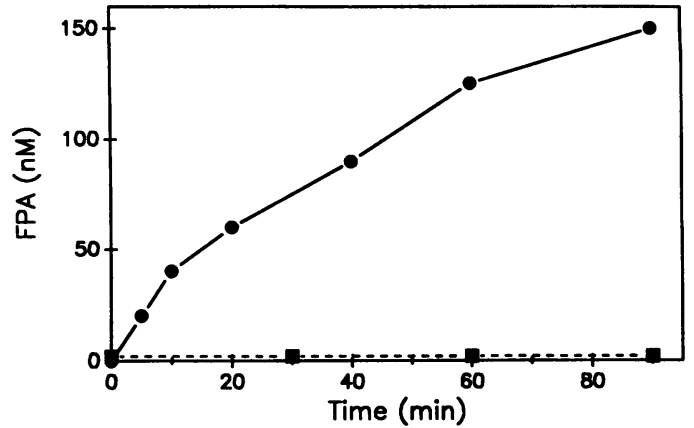

Figure 3. Time-course of FPA generation by thrombin bound to fibrin. Citrated plasma was incubated for $90 \mathrm{~min}$ at $37^{\circ} \mathrm{C}$ in the presence (solid line) or absence (broken line) of a washed fibrin clot. At the times indicated, aliquots of plasma were removed, unreacted fibrinogen was precipitated with ethanol, and the ethanol supernatants were assayed for FPA.

increase does not result from the release of trapped peptide since $<5$ nM FPA is recovered when clots are incubated in buffer in place of plasma. Second, clot-bound thrombin is responsible for this activity since no FPA is generated when clots preincubated for $30 \mathrm{~min}$ at $37^{\circ} \mathrm{C}$ in buffer containing 10 $\mathrm{nM}$ hirudin or $6 \mathrm{nM}$ PPACK are washed and then incubated in plasma. Third, the amount of FPA generated by clot-bound thrombin is directly related to clot size (Table I), consistent with the concept that thrombin binding is dependent on the amount of available fibrin. In addition, the amount of FPA generated by clot sonicates is approximately threefold higher than that produced by intact clots (Table I). This finding suggests that only thrombin bound to the surface of intact clots is available to interact with fibrinogen, whereas additional thrombin is exposed in the clot sonicates.

Incubation of clot sonicates with citrated plasma results in progressive FPA generation with a time course similar to that produced by the intact clots (data not shown). These findings suggest that thrombin that remains bound to clot fragments is also protected from fluid-phase antiproteinases.

Comparison of the activity of thrombin inhibitors against fluid-phase and clot-bound thrombin. To determine the ability of thrombin inhibitors to inactivate thrombin bound to fibrin, clots were incubated in plasma in the presence or absence of the various agents and FPA release was quantified. These results were then compared with the activity of the thrombin inhibitors against FPA generated by fluid-phase thrombin.

Table I. FPA Generation at 60-min Incubation by Intact and Sonicated Clots of Different Sizes

\begin{tabular}{|c|c|c|c|}
\hline \multirow[b]{2}{*}{ Clot mass } & \multicolumn{2}{|c|}{ FPA } & \multirow[b]{2}{*}{ Ratio } \\
\hline & Intact & Sonicated & \\
\hline$c p m \times 10^{-3}$ & \multicolumn{2}{|c|}{$n M$} & \\
\hline 28.9 & 41.0 & 131.8 & 3.2 \\
\hline 62.3 & 92.5 & 249.5 & 2.7 \\
\hline 94.5 & 117.1 & 359.6 & 3.8 \\
\hline 126.7 & 160.8 & 478.3 & 2.8 \\
\hline
\end{tabular}

The results shown represent the mean of three separate experiments. 


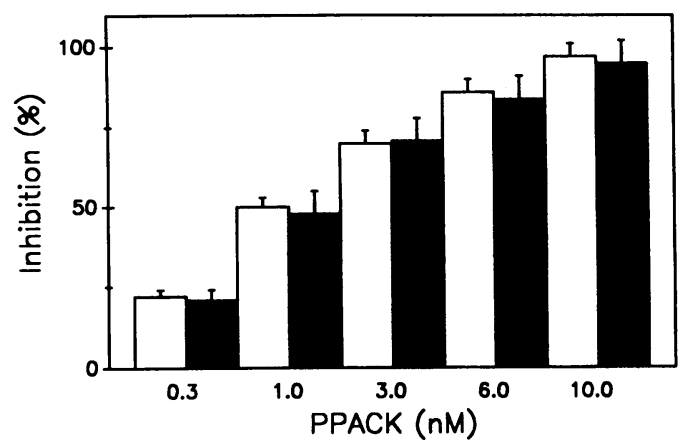

Figure 4. Comparison of the inhibitory effect of PPACK against fluid-phase (open bars) and clot-bound thrombin activity (solid bars). To determine the inhibitory effect against fluid-phase thrombin activity, $\alpha$-thrombin $(0.2-4.0 \mathrm{nM})$ was incubated with citrated plasma for $60 \mathrm{~min}$ at $37^{\circ} \mathrm{C}$ in the absence or presence of PPACK at the concentrations indicated. At the end of the incubation period, the plasma levels of FPA were determined, and the percent inhibition of FPA generation was then calculated for each inhibitor concentration. To determine the inhibitory effect against clot-bound thrombin activity, washed fibrin clots were incubated in citrated plasma for 60 $\min$ at $37^{\circ} \mathrm{C}$ in the absence or presence of varying concentrations of PPACK. At the end of the incubation period, the plasma levels of FPA were determined, and the percent inhibition of clot-induced FPA generation was then calculated for each inhibitor concentration. Each bar represents the mean of three separate experiments (each done in duplicate), while the lines above the bars represent the SD.

PPACK (Fig. 4) and hirugen (Fig. 5) are equally effective inhibitors of fluid-phase and clot-bound thrombin because they block FPA release mediated by free thrombin and by the clotbound enzyme to a similar extent. Hirudin is slightly less active against solid-phase thrombin than it is against the fluidphase enzyme since each concentration of this agent inhibits the activity of clot-bound thrombin $50 \%$ less than the fluidphase enzyme (Fig. 6). In contrast, heparin is much less effective at inhibiting thrombin bound to fibrin (Fig. 7). For example, $0.1 \mathrm{U} / \mathrm{ml}$ of heparin produces $70 \%$ inhibition of FPA release mediated by fluid-phase thrombin but has almost no activity against the clot-bound enzyme. Although heparin concentrations of $0.2-0.4 \mathrm{U} / \mathrm{ml}$, which span the therapeutic

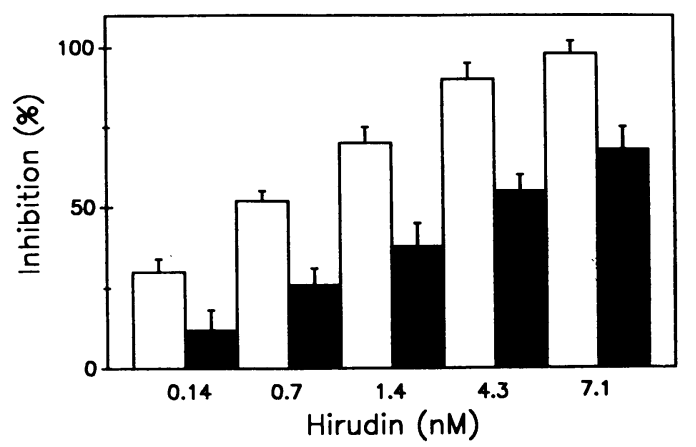

Figure 6. Comparison of the inhibitory effect of hirudin against fluid-phase (open bars) and clot-bound thrombin activity (solid bars). The inhibitory activity of hirudin was determined as described in the legend to Fig. 4. Each bar represents the mean of three separate experiments (each done in duplicate), while the lines above the bars represent the SD.

range for this agent (30), completely inhibit FPA release mediated by free thrombin, they produce only $20-40 \%$ inhibition of clot-bound FPA generation. Similarly, a concentration of 1 $\mathrm{U} / \mathrm{ml}$, which may be achieved after $5,000 \mathrm{U}$ of heparin is given as a bolus to patients (31), inhibits the activity of clot-bound thrombin by only $60 \%$. Increased inhibition is produced by higher concentrations of heparin, and a concentration of 2 $\mathrm{U} / \mathrm{ml}$ produces $70 \%$ inhibition, an amount equivalent to the inhibition of fluid-phase thrombin effected by a concentration of heparin of $0.1 \mathrm{U} / \mathrm{ml}$.

Inability of thrombin inhibitors to displace ${ }^{125}$ I-labeled thrombin bound to fibrin. To directly monitor thrombin binding to fibrin we measured the incorporation of ${ }^{125}$ I-labeled thrombin into clots. After aging the clots for $60 \mathrm{~min}$ and washing them thoroughly to remove trapped thrombin, $29.2 \pm 3.5 \%$ of the labeled enzyme was bound to fibrin. Despite $18 \mathrm{~h}$ of extensive washing at $23^{\circ} \mathrm{C}, 10.4 \pm 1.8 \%$ of the ${ }^{125} \mathrm{I}$-labeled thrombin remained bound to fibrin. The ability of the various thrombin inhibitors to displace bound thrombin was then tested. Using each of the inhibitors at the concentration that produced $70 \%$ inhibition of FPA generation by clotbound thrombin (i.e., $2.0 \mathrm{U} / \mathrm{ml}$ heparin, $7.1 \mathrm{nM}$ hirudin, 1.9

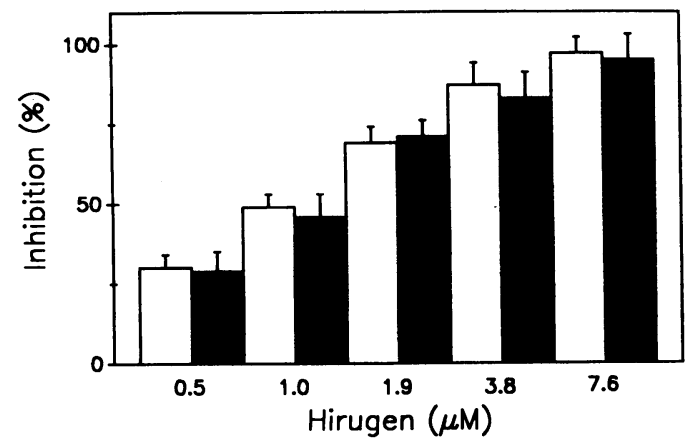

Figure 5. Comparison of the inhibitory effect of hirugen against fluid-phase (open bars) and clot-bound thrombin activity (solid bars). The inhibitory activity of hirugen was determined as described in the legend to Fig. 4. Each bar represents the mean of three separate experiments (each done in duplicate), while the lines above the bars represent the SD.

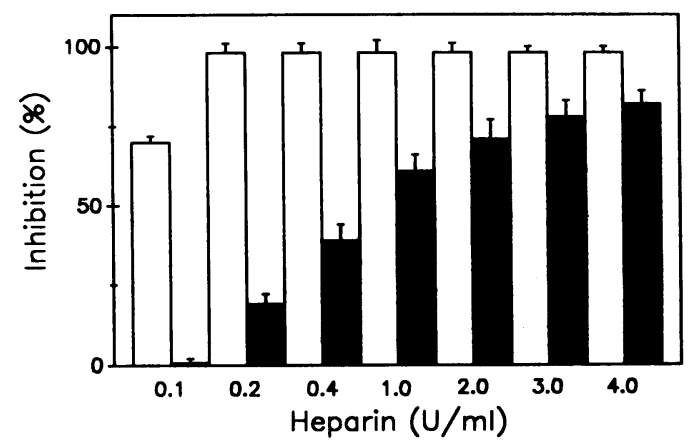

Figure 7. Comparison of the inhibitory effect of heparin against fluid-phase (open bars) and clot-bound thrombin activity (solid bars). The inhibitory activity of heparin was determined as described in the legend to Fig. 4. Each bar represents the mean of three separate experiments (each done in duplicate), while the lines above the bars represent the SD. 
$\mu \mathrm{M}$ hirugen, and $3 \mathrm{nM}$ PPACK), displacement of ${ }^{125}$ I-labeled enzyme bound to the clot was not observed.

\section{Discussion}

These studies confirm previous reports that thrombin bound to fibrin is enzymatically active (2-7) and is protected from inhibition by heparin-antithrombin III (12). However, we have extended these observations in a number of important ways. First, we have demonstrated that clot-bound thrombin is capable of releasing FPA from fibrinogen despite the presence of physiologic concentrations of antiproteinases (Fig. 3) since our studies were done in plasma and not in a buffer system. Second, we have shown that thrombin bound to fibrin polymer is relatively protected from heparin inactivation (Fig. 7). The use of plasma-derived clots rather than artificially produced fibrin monomer (12) models more closely the physiologic events that occur during the coagulation process. Third, we have compared the susceptibility to inactivation of thrombin bound to fibrin with that of thrombin free in solution. These studies indicate that clot-bound thrombin is relatively protected from inactivation by heparin but is susceptible to inhibition by antithrombin III-independent inhibitors.

The thrombin inhibitors used in our study are heparin, hirudin, hirugen, and PPACK. These agents were chosen because they have different mechanisms of action. Heparin catalyzes thrombin inactivation by antithrombin III (8-11), while the other thrombin inhibitors act independently of antithrombin III. Hirudin, a potent and specific inhibitor derived from Hirudo medicinalis, forms an essentially irreversible, stoichiometric complex with thrombin with a dissociation constant reported as low as $20 \mathrm{fM}$ (32). Recombinant forms of hirudin also have potent antithrombotic activity despite the fact that they are not sulfated at Tyr-63 (33). Hirugen is a synthetic Tyr-sulfated dodecapeptide comprising residues 53-64 of the carboxy-terminal region of hirudin (19). The antithrombin activity of hirugen appears to be the result of interaction with a noncatalytic site on thrombin, since hirugen blocks the fibrinogenolytic activity of the enzyme without affecting the amidolytic activity of thrombin against low molecular weight synthetic substrates (21). In contrast, PPACK binds to the active site of thrombin, wherein the active center histidine is irreversibly alkylated by the chloromethyl ketone (34).

We compared the ability of these thrombin inhibitors to block fluid-phase thrombin with their activity against thrombin bound to fibrin. For both systems, plasma levels of FPA were used as an index of unopposed thrombin activity. The addition of $\alpha$-thrombin to citrated plasma results in concentration-dependent generation of FPA (Fig. 1). With each concentration of thrombin, FPA release reaches a plateau within minutes, consistent with rapid enzyme inhibition by plasma antithrombins. All of the thrombin inhibitors produce concentration-dependent inhibition of thrombin-mediated FPA release in plasma. Using the plasma system, we then identified concentrations of each agent that produce approximately equivalent inhibition of FPA release (Fig. 2).

The time course of FPA generation by clot-bound thrombin (Fig. 3) is different from that produced by the fluid-phase enzyme (Fig. 1). In the presence of a clot that has been thoroughly washed to remove trapped FPA and unbound throm- bin, there is progressive FPA generation throughout the incubation period, indicating that clot-bound thrombin is enzymatically active and is protected from inactivation by plasma antiproteinases. Clots of increasing size generate more FPA (Table I), presumably because the larger surface area exposes more thrombin to fibrinogen. Sonicates of washed clots generate approximately threefold more FPA than do intact clots, suggesting that fibrinogen in the surrounding plasma does not have free access to thrombin bound to fibrin in the interstices of the intact clot.

The ability of thrombin inhibitors to block FPA release mediated by fluid-phase thrombin was then compared with their activity against the clot-bound enzyme. Heparin is a relatively poor inhibitor of thrombin bound to fibrin since a concentration of heparin that inhibits $\sim 70 \%$ of thrombin-mediated FPA release has no effect on the activity of the clotbound enzyme (Fig. 7). Instead, a 20-fold higher heparin concentration is required to produce equivalent inhibition of clot-induced FPA release $(2.0$ and $0.1 \mathrm{U} / \mathrm{ml}$, respectively). Therefore, like thrombin bound to fibrin monomer (12), enzyme bound to a clot is also relatively protected from inactivation by heparin-antithrombin III. In contrast, PPACK and hirugen are equally effective inhibitors of fluid- and solidphase thrombin (Figs. 4 and 5, respectively). Hirudin is a slightly better inhibitor of the fluid-phase enzyme since each concentration of this agent produces twofold more inhibition of free thrombin than of the clot-bound enzyme (Fig. 6). These findings suggest that clot-bound thrombin may be readily accessible to low molecular weight inhibitors such as hirugen and PPACK (1,578 and $524 \mathrm{D}$, respectively), and less susceptible to inhibition by the 7,000-D hirudin molecule.

To explore the mechanism by which thrombin bound to fibrin is protected from inhibition by heparin-antithrombin III but susceptible to inactivation by antithrombin III-independent inhibitors, we examined the ability of the various agents to displace ${ }^{125}$ I-labeled thrombin from the clot. When used at concentrations that blocked $70 \%$ of clot-induced FPA generation (i.e., $0.1 \mathrm{U} / \mathrm{ml}$ heparin, $1.4 \mathrm{nM}$ hirudin, $1.9 \mu \mathrm{M}$ hirugen, and 3.0 nM PPACK), none of the inhibitors displaced fibrin-bound thrombin into the surrounding medium. PPACK, which blocks the active center histidine of thrombin (34), would not be expected to displace thrombin since the enzyme binds to fibrin through a site distinct from its catalytic center (2-7). Hirudin binds to two sites on thrombin during the formation of the enzyme-inhibitor complex. One is a low affinity site that does not impair thrombin-catalyzed hydrolysis of low molecular weight substrates, while the other is a high affinity site near the catalytic center of the enzyme (32). Hirugen is an analogue of the carboxy-terminal region of hirudin. Its antithrombin effect is thought to be the result of hirugen interaction with the noncatalytic fibrinogen binding site on thrombin (21). The experiments with ${ }^{125} \mathrm{I}$-labeled thrombin suggest that inhibition of clot-bound thrombin by hirugen and hirudin does not depend on enzyme displacement from its fibrin binding site.

Other investigators have demonstrated that hirudin can displace thrombin that is bound to fibrin monomer-Sepharose $(4,6)$. Our results are not necessarily incompatible with these findings since we investigated the displacement of thrombin bound to fibrin clots rather than to fibrin monomer. In addition, we used concentrations of hirudin that effectively block 
FPA generation by clot-bound thrombin. Although these concentrations of hirudin do not displace the enzyme from fibrin, they are $\sim 400$-fold lower than the concentrations necessary to displace thrombin from fibrin monomer-Sepharose $(4,6)$.

Heparin acts as an anticoagulant principally by catalyzing antithrombin III-mediated inactivation of thrombin (8-11). To accomplish this, heparin acts as a template and binds to both thrombin and antithrombin III $(10,11)$. Recent studies in buffer systems have demonstrated that thrombin bound to fibrin monomer is protected from inactivation by heparin-antithrombin III (12). Our findings are consistent with these observations, and demonstrate that thrombin bound to fibrin clots is relatively protected from inhibition by heparin-antithrombin III in a plasma system. In addition, it is likely that the heparin binding site on thrombin is inaccessible when the enzyme is bound to fibrin, since concentrations of heparin that produce $70 \%$ inhibition of FPA release mediated by fluidphase thrombin do not inhibit the clot-bound enzyme. A recent study by Mirshahi et al. (35) suggests that thrombin bound to fibrin degradation products also may be relatively protected from inactivation by heparin since enzyme released from the clot during pharmacologically induced fibrinolysis is more susceptible to inactivation by hirudin than by heparin.

Although heparin has proven to be an extremely effective antithrombotic agent, both experimental and clinical studies indicate that it is limited in its ability to inhibit the propagation of venous thrombi (14-18) or to prevent coronary rethrombosis after thrombolytic therapy $(19,20)$. The studies presented here provide a plausible explanation for these limitations. Further, they raise the possibility that antithrombin IIIindependent inhibitors may be more effective than heparin in these clinical settings.

\section{Acknowledgments}

The authors thank Dr. Michael Nesheim for helpful discussions and Susan Crnic for preparing this manuscript.

This work was supported by grants from the Medical Research Council of Canada and the Ontario Heart and Stroke Foundation. Dr. Weitz is a Scholar of the Heart and Stroke Foundation of Ontario.

\section{References}

1. Blomback, B., B. Hessel, D. Hogg, and L. Therkildsen. 1978. A two-step fibrinogen-fibrin transition in blood coagulation. Nature (Lond.). 275:501-505.

2. Seegers, W. H., M. Nieff, and J. A. Shafer. 1945. Note on the adsorption of thrombin on fibrin. Science (Wash. DC). 101:520-521.

3. Liu, C. Y., H. L. Nossel, and K. L. Kaplan. 1979. The binding of thrombin by fibrin. J. Biol. Chem. 254:10421-10425.

4. Wilner, G. D., M. P. Danitz, M. S. Mudd, K. H. Hsieh, and J. W. Fenton II. 1981. Selective immobilization of thrombin by surface bound fibrin. J. Lab. Clin. Med. 97:403-411.

5. Berliner, L. J., Y. Sugawara, and J. W. Fenton II. 1985. Human $\alpha$-thrombin binding to nonpolymerized fibrin-Sepharose: Evidence for an anionic binding region. Biochemistry. 24:7005-7009.

6. Kaminski, M., and J. McDonagh. 1987. Inhibited thrombins: interactions with fibrinogen and fibrin. Biochem. J. 242:881-887.

7. Vali, Z., and H. A. Scheraga. 1988. Localization of the binding site on fibrin for the secondary binding site of thrombin. Biochemistry. 27:1956-1963.

8. Rosenberg, R. D., and P. S. Damus. 1973. The purification and mechanism of action of human antithrombin-heparin cofactor. $J$. Biol. Chem. 248:6490-6505.

9. Bjork, I., and U. Lindahl. 1982. Mechanism of the anticoagulant action of heparin. Mol. Cell. Biochem. 48:161-182.

10. Danielsson, A., E. Raub, U. Lindahl, and I. Bjork. 1986. Role of ternary complexes, in which heparin binds both antithrombin and proteinase, in the acceleration of the reactions between antithrombin and thrombin or factor Xa. J. Biol. Chem. 261:15467-15473.

11. Olson, S. T. 1988. Transient kinetics of heparin-catalyzed protease inactivation by antithrombin III. Linkage of protease-inhibitorheparin interactions in the reaction with thrombin. J. Biol. Chem. 263:1698-1708.

12. Hogg, P. J., and C. M. Jackson. 1989. Fibrin monomer protects thrombin from inactivation by heparin-antithrombin III: implications for heparin efficacy. Proc. Natl. Acad. Sci. USA. 86:3619-3623.

13. Boneu, B., M. R. Buchanan, J. F. Cade, J. Van Ryn, F. F. Fernandez, F. A. Ofosu, and J. Hirsh. 1985. Effects of heparin, its low molecular weight fractions and other glycosaminoglycans on thrombus growth in vivo. Thromb. Res. 40:81-89.

14. Marder, V. J., R. L. Soulen, V. Atichartakarn, A. Z. Budzynski, S. Parulekar, J. R. Kim, N. Edward, J. Zahavi, and K. M. Algazy. 1977. Quantitative venographic assessment of deep vein thrombosis in the evaluation of streptokinase and heparin therapy. J. Lab. Clin. Med. 89:1018-1029.

15. Bratt, G., E. Tornebohm, S. Gragvist, W. Aberg, and D. Lockner. 1985. A comparison between low molecular weight heparin (KABI 2165) and standard heparin in the intravenous treatment of deep vein thrombosis. Thromb. Haemostasis. 54:813-817.

16. Holm, H. A., B. Ly, G. F. Handeland, U. Abildgaard, K. E. Arnesen, P. Gottschalk, V. Hoeg, M. Aandahl, K. Haugen, F. Laerum, B. Scheel, O. Sortland, and B. Vinje. 1986. Subcutaneous heparin treatment of deep vein thrombosis: a comparison of unfractionated and low molecular weight heparin. Haemostasis. 16 (Suppl. 2):30-37.

17. Lagerstedt, C. I., B. O. Fagher, U. Albrechtsson, C. G. Olsson, and B. W. Oqvist. 1985. Need for long-term anticoagulant treatment in symptomatic calf-vein thrombosis. Lancet. i:515-518.

18. Hull, R., T. Delmore, E. Genton, J. Hirsh, M. Gent, D. Sackett, D. McLoughlin, and P. Armstrong. 1979. Warfarin sodium versus low-dose heparin in the long-term treatment of venous thrombosis. $N$. Engl. J. Med. 301:855-858.

19. Yasuda, T., H. K. Gold, J. T. Fallon, R. C. Leinbach, J. L. Guerrero, L. E. Scudder, M. Kanke, D. Shealy, M. J. Ross, D. Collen, and B. S. Coller. 1988. Monoclonal antibody against the platelet glycoprotein (GP) IIb/IIIa receptor prevents coronary artery reocclusion after reperfusion with recombinant tissue-type plasminogen activator in dogs. J. Clin. Invest. 81:1284-1291.

20. Topol, E. J., B. S. George, D. J. Kereiakes, D. C. Stump, R. J. Candela, C. W. Abbotsmith, L. Aronson, A. Pickel, J. M. Boswick, K. L. Lee, et al. 1989. A randomized controlled trial of intravenous plasminogen activator and early intravenous heparin in acute myocardial infarction. Circulation. 79:281-286.

21. Maraganore, J., B. Chao, M. L. Joseph, J. Jablonski, and K. L. Ramachandran. 1989. Anticoagulant activity of synthetic hirudin peptides. J. Biol. Chem. 264:8692-8698.

22. Nossel, H. L., I. Yudelman, R. E. Canfield, V. P. Butler, Jr., K. Spanondis, G. D. Wilner, and G. D. Qureshi. 1974. Measurement of fibrinopeptide A in human blood. J. Clin. Invest. 54:43-53.

23. Nossel, H. L., V. P. Butler, Jr., G. D. Wilner, R. E. Canfield, and E. J. Harfenist. 1976. Specificity of antisera to human fibrinopeptide A used in clinical fibrinopeptide A assays. Thromb. Haemostasis. 35:101-109.

24. Canfield, R. E., J. Dean, H. L. Nossel, V. P. Butler, Jr., and G. D. Wilner. 1976. Reactivity of fibrinogen and fibrinopeptide A containing fragments with antisera to fibrinopeptide A. Biochemistry. 15:1203-1209.

25. Weitz, J. I., S. L. Landman, K. A. Crowley, S. Birken, and F. J. Morgan. 1986. Development of an assay for in vivo human neutrophil 
elastase activity. Increased elastase activity in patients with $\alpha_{1}$-proteinase inhibitor deficiency. J. Clin. Invest. 78:155-162.

26. Weitz, J. I., M. K. Cruickshank, B. Thong, B. Leslie, M. N. Levine, and T. Eckhardt. 1988. Human tissue-type plasminogen activator releases fibrinopeptides A and B from fibrinogen. J. Clin. Invest. 82:1700-1707.

27. Ikeno, L. C., B. M. Bowen, and M. Der. 1981. Commercial production of ${ }^{125} \mathrm{I}$-fibrinogen injection. J. Radioanal. Chem. 65:179188.

28. McFarlane, A. S. 1956. Labelling of plasma proteins with radioactive iodine. Biochem. J. 62:135-143.

29. Martin, B. M., W. W. Wasiewski, J. W. Fenton II, and T. C. Detwiler. 1976. Equilibrium binding of thrombin to platelets. Biochemistry. 15:4886-4893.

30. Hull, R. D., G. E. Raskob, J. Hirsh, R. M. Jay, J. R. LeClerc, W. H. Geerts, D. Rosenbloom, D. L. Sackett, C. Anderson, L. Harrison, and M. Gent. 1986. Continuous intravenous heparin compared with intermittent subcutaneous heparin in the initial treatment of proximal vein thrombosis. N. Engl. J. Med. 315:1109-1114.

31. Hirsh, J., W. G. van Arken, A. S. Gallus, C. G. Dollery, J. S. Cade, and W. G. Young. 1976. Heparin kinetics in venous thrombosis and pulmonary embolism. Circulation. 53:691-695.

32. Stone, S. R., and J. Hofsteenge. 1986. Kinetics of the inhibition of thrombin by hirudin. Biochemistry. 25:4622-4628.

33. Talbot, M. D., J. Ambler, K. D. Butler, V. S. Findlay, K. A. Mitchell, R. F. Peters, M. F. Tweed, and R. B. Wallis. 1989. Recombinant desulphatohirudin (CGP 39393) anticoagulant and antithrombotic properties in vivo. Thromb. Haemostasis. 61:77-80.

34. Kettner, C., and E. Shaw. 1979. D-Phe-Pro-Arg $\mathrm{CH}_{2} \mathrm{Cl}$. A selective affinity label for thrombin. Thromb. Res. 14:969-973.

35. Mirshahi, M., J. Soria, C. Soria, F. Faivre, H. Lu, M. Courtney, C. Roitsch, D. Tripier, and J. P. Caen. 1989. Evaluation of the inhibition by heparin and hirudin of coagulation activation during rt-PA-induced thrombolysis. Blood. 74:1026-1030. 